Modern Physics Letters A

(C) World Scientific Publishing Company

\title{
DECAY PROBABILITY RATIO OF PENTAQUARK $\Theta^{+}$STATE
}

\author{
XUN CHEN \\ School of Physics, Peking University, Beijing 100871, China \\ YAJUN MAO \\ School of Physics, Peking University, Beijing 100871, China \\ BO-QIANG MA* \\ School of Physics, Peking University, Beijing 100871, China
}

Receivd (Day Month Year)

Revised (Day Month Year)

\begin{abstract}
The pentaquark state of $\Theta^{+}(u u d d \bar{s})$ has been observed to decay with two decay modes: $\Theta^{+} \rightarrow n K^{+}$and $\Theta^{+} \rightarrow p K^{0}$. The decay probability ratio of the two decay modes is studied with general symmetry consideration of isospin, spin, and parity. We arrive at a result of the ratio $\frac{\Gamma\left(\Theta^{+} \rightarrow n K^{+}\right)}{\Gamma\left(\Theta^{+} \rightarrow p K^{0}\right)}=\frac{(\alpha-\beta)^{2}}{(\alpha+\beta)^{2}}\left(\frac{k_{1}}{k_{2}}\right)^{2 L+1}$, which is valid for the $\Theta^{+}$state to be a pure isoscalar or isovector state, or an isoscalar and isovector mixing state, or an isotensor state with mixture of isoscalar and isovector components with coefficients $\alpha$ and $\beta$. The dependence on spin and parity of the pentaquark $\Theta^{+}$state is found to be small due to small difference between the center of mass decay momenta $k_{1}$ and $k_{2}$ of the two decay modes. We also provide an analysis on the constraint of the isospin of $\Theta^{+}$from the absence of a peak in the $p K^{+}$invariant mass distribution in the $\gamma p \rightarrow p K^{+} K^{-}$process. Future experimental results about the decay probability ratio may provide information about the properties of the pentaquark $\Theta^{+}$state.
\end{abstract}

Keywords: pentaquark; isospin; spin and parity; hadronic decays

PACS Nos.: 12.39.Mk; 11.30.-j; 11.30.Er; 13.30.Eg

The standard quark mode 1 is remarkably successful in classifying hadrons as composite systems of quark-antiquark $(q \bar{q})$ states for mesons and three quark $(q q q)$ states for baryons. The possible existence of multiquark states with four quarks and an antiquark, i.e., the pentaquark states with configuration $q q q q \bar{q}, 2314$ has been discussed for more than two decades. QCD based models permit the existence of pentaquark states. ${ }^{[2]}$ The pentaquark states with the antiquark $\bar{q}$ being a heavy quark are suggested to exist. 3 Earlier experimental attempts were focused on searching for such pentaquark states containing at least one heavy flavor quark (antiquark), but without convincing evidence. ${ }^{[5}$ It is noticed that $q q q q \bar{q}$ is unambiguously a minimal pentaquark state if the flavor of $\bar{q}$ is different from any of the other four quarks!

*Corresponding author. Email address: mabq@phy.pku.edu.cn 
The possible existence of the minimal pentaquark states with the antiquark being an anti-strange quark $\bar{s}$, such as $\Theta^{+}(u u d d \bar{s})$ and $\Theta^{++}(u u u d \bar{s})$, are discussed in Ref. 4. with suggestion for experimental searches also provided.

From another point of view, baryons can be viewed as solitons in an effective meson theory $\left[6\right.$ as well as in the large $N_{c^{-}}$-limit of QCD ${ }^{[7}$ Thus baryons can be also classified by soliton configurations in chiral field theories, as an alternative scheme compared to the standard quark model classification. In the $\mathrm{SU}(3)$ version of these models, the anti-decuplet $\{\overline{10}\}$ baryon multiplet is the lowest multiplet above the minimal octet $\{8\}$ and decuplet $\{10\}$ baryons $\frac{8}{8}$ It is found $\frac{9101112}{S}$ that there exists a baryon state with strangeness $S=+1$, which can be identified with the pentaquark $\Theta^{+}(u u d d \bar{s})$ configure, in this $\{\overline{10}\}$ multiplet. By treating the experimentally observed N(1710) resonance as a member of this multiplet, Diakonov, Petrov, and Polyakov predicted 11 the $\Theta^{+}$baryon state to have a mass of about $1530 \mathrm{MeV}$ and a width of about $15 \mathrm{MeV}$. This baryon state is nothing exotic compared to other baryons in the soliton classification scheme, except that it has a strangeness number $S=+1$ and has also a surprising narrow width.

The recent observations of an exotic narrow baryon state with $S=+1$ by LEPS, 13 DIANA, 14 CLAS, 15 SAPHIR, 16 and HERMES 17 Collaborations, provide evidence for the existence of the pentaquark state $\Theta^{+}(u u d d \bar{s})$. The detection of the $\Theta^{+}$state in LEPS and CLAS experiments is through the subprocess $\gamma n \rightarrow K^{-}\left(K^{+} n\right)$, where $K^{-}$and $K^{+}$are detected with the $\Theta^{+}$state constructed from the missing mass of the $K^{+} n$ system, $\frac{18}{18}$ a same principle to the $\gamma^{*} n \rightarrow K^{-} \Theta^{+}$process suggested in Ref. 4 The SAPHIR experiment detects the $\Theta^{+}(u u d d \bar{s})$ state through the process $\gamma p \rightarrow n K_{s}^{0} K^{+}$. In DIANA and HERMES experiments, the $\Theta^{+}$state is detected through the decay mode $\Theta^{+} \rightarrow K^{0} p$, where the $K^{0}$ meson is constructed by the decay $K_{s}^{0} \rightarrow \pi^{+} \pi^{-}$. All of the experiments have observed a sharp peak in the $K^{+} n$ missing mass spectrum or in the $K_{s}^{0} p$ invariant mass spectrum, with a mass around $1540 \mathrm{MeV}$ and a narrow width around $9 \rightarrow 25 \mathrm{MeV}$, in agreement with the $\Theta^{+}$state predicted by the chiral quark soliton model 11 Therefore two decay modes: $\Theta^{+} \rightarrow n K^{+}$and $\Theta^{+} \rightarrow p K^{0}$, have been observed for the $\Theta^{+}$state.

The properties of the observed $\Theta^{+}$state, such as its spin, isospin, and parity, are still not established yet. The chiral quark soliton model predicts this $\Theta^{+}$state of having spin $1 / 2$, isospin 0 , and parity + of the anti-decuplet ${ }^{11}$ However, with a mass of $100 \mathrm{MeV}$ above threshold, this pentaquark state should be expected to have a decay width of the order of $500 \mathrm{MeV}$ unless its decays are suppressed by phase space, symmetry, or special dynamics ${ }^{19}$ It is suggested by Capstick, Page, and Roberts $\frac{19}{19}$ that the narrow width of the observed $\Theta^{+}$state can be understood if the isospin of this state is hypothesized to be 2, i.e., an isotensor resonance. For this isospin, the decays of $\Theta^{+} \rightarrow n K^{+}$and $\Theta^{+} \rightarrow p K^{0}$ via isospin violating hadronic processes are suppressed by symmetry. Jaffe and Wilczek tried to explain the narrow width by proposing the $\Theta^{+}$state, which is an isospin singlet, as being composed of two highly correlated $u d$-pairs and an $\bar{s}$ quark ${ }^{20}$ This work intends to 
study the probability ratio of the two decay modes, and to show that this ratio can provide useful information on the properties of the observed $\Theta^{+}$state.

Our analysis is based on general symmetry consideration of isospin $\left(I, I_{z}\right)$, spin $J$, and parity $P$. The corresponding quantum numbers of quarks are

$$
\left(I, I_{z}\right)\left(J^{p}\right)= \begin{cases}\left(\frac{1}{2},+\frac{1}{2}\right)\left(\frac{1}{2}^{+}\right), & \text {for the } u \text { quark; } \\ \left(\frac{1}{2},-\frac{1}{2}\right)\left(\frac{1}{2}^{+}\right), & \text {for the } d \text { quark; } \\ (0,0)\left(\frac{1}{2}^{+}\right), & \text {for the } s \text { quark. }\end{cases}
$$

The pentaquark $\Theta^{+}(u u d d \bar{s})$ state should have isospin $I=0,1$ or 2 with $I_{z}=0$, therefore we can obtain the general isospin configuration of this state

$$
\left|\Theta^{+}\right\rangle=a\left|I=2, I_{z}=0\right\rangle+\alpha|1,0\rangle+\beta|0,0\rangle,
$$

where $a=\sqrt{1-\alpha^{2}-\beta^{2}}$. The proton $p$ state should have isospin $I=1 / 2$ with $I_{z}=1 / 2$, and the neutron $n$ state should have isospin $I=1 / 2$ with $I_{z}=-1 / 2$. If the nucleon state contains a small isospin impurities, we have

$$
\begin{gathered}
|p\rangle=b\left|\frac{1}{2}, \frac{1}{2}\right\rangle+\gamma\left|\frac{3}{2}, \frac{1}{2}\right\rangle, \\
|n\rangle=b\left|\frac{1}{2},-\frac{1}{2}\right\rangle+\gamma\left|\frac{3}{2},-\frac{1}{2}\right\rangle,
\end{gathered}
$$

where $b=\sqrt{1-\gamma^{2}}$, and $\gamma$ is a very small quantity refelcting isospin violating!2122 The kaon $K^{+}$state should have isospin $I=1 / 2$ with $I_{z}=1 / 2$, and the kaon $K^{0}$ state should have isospin $I=1 / 2$ with $I_{z}=-1 / 2$. Thus we have

$$
\begin{aligned}
& \left|K^{+}\right\rangle=\left|\frac{1}{2}, \frac{1}{2}\right\rangle, \\
& \left|K^{0}\right\rangle=\left|\frac{1}{2},-\frac{1}{2}\right\rangle .
\end{aligned}
$$

The $n K^{+}$final state can be written as

$$
\left|n, K^{+}\right\rangle=b\left|\frac{1}{2}-\frac{1}{2}, \frac{1}{2} \frac{1}{2}\right\rangle+\gamma\left|\frac{3}{2}-\frac{1}{2}, \frac{1}{2} \frac{1}{2}\right\rangle,
$$

and the $p K^{0}$ state can be written as

$$
\left|p, K^{0}\right\rangle=b\left|\frac{1}{2} \frac{1}{2}, \frac{1}{2}-\frac{1}{2}\right\rangle+\gamma\left|\frac{3}{2} \frac{1}{2}, \frac{1}{2}-\frac{1}{2}\right\rangle .
$$

Isospin is conserved in hadronic decays via strong interaction, thus the $\Theta^{+}$state can not decay into $n K^{+}$and $p K^{0}$ final states if $\Theta^{+}$is a pure $I=2$ isotensor state. Assuming that $\alpha$ and $\beta$ are of the same size as the mixing coefficient of 0.015 estimated from the physical $\Lambda$ and $\Sigma^{0}$ mixing, 23 a narrow width of the $\Theta^{+}$state can be understood 19 We will show in the following that the difference between $\alpha$ and $\beta$ can introduce difference between the decay probabilities of the two decay modes for the $\Theta^{+}$state. 
We may expand the $\Theta^{+}$state Eq. (2) by using Eqs. (3) and (4),

$$
\begin{aligned}
& a|2,0\rangle+\alpha|1,0\rangle+\beta|0,0\rangle \\
\simeq & a \gamma\left|\frac{3}{2}-\frac{1}{2}, \frac{1}{2} \frac{1}{2}\right\rangle\left\langle\frac{3}{2}-\frac{1}{2}, \frac{1}{2} \frac{1}{2} \mid 2,0\right\rangle \\
& +a \gamma\left|\frac{3}{2} \frac{1}{2}, \frac{1}{2}-\frac{1}{2}\right\rangle\left\langle\frac{3}{2} \frac{1}{2}, \frac{1}{2}-\frac{1}{2} \mid 2,0\right\rangle \\
& +b \alpha\left|\frac{1}{2}-\frac{1}{2}, \frac{1}{2} \frac{1}{2}\right\rangle\left\langle\frac{1}{2}-\frac{1}{2}, \frac{1}{2} \frac{1}{2} \mid 1,0\right\rangle \\
& +b \alpha\left|\frac{1}{2} \frac{1}{2}, \frac{1}{2}-\frac{1}{2}\right\rangle\left\langle\frac{1}{2} \frac{1}{2}, \frac{1}{2}-\frac{1}{2} \mid 1,0\right\rangle \\
& \left.+b \beta \frac{1}{2}-\frac{1}{2}, \frac{1}{2} \frac{1}{2}\right\rangle\left\langle\frac{1}{2}-\frac{1}{2}, \frac{1}{2} \frac{1}{2} \mid 0,0\right\rangle \\
& +b \beta\left|\frac{1}{2} \frac{1}{2}, \frac{1}{2}-\frac{1}{2}\right\rangle\left\langle\frac{1}{2} \frac{1}{2}, \frac{1}{2}-\frac{1}{2} \mid 0,0\right\rangle,
\end{aligned}
$$

in which the $\alpha \gamma$ term is ignored. On the other hand, we have the Clebsch-Gorden coefficients for the isospin summation

$$
\begin{aligned}
& \left\langle\frac{3}{2}-\frac{1}{2}, \frac{1}{2} \frac{1}{2} \mid 2,0\right\rangle=1 / \sqrt{2}, \quad\left\langle\frac{3}{2} \frac{1}{2}, \frac{1}{2}-\frac{1}{2} \mid 2,0\right\rangle=1 / \sqrt{2} \\
& \left\langle\frac{1}{2}-\frac{1}{2}, \frac{1}{2} \frac{1}{2} \mid 1,0\right\rangle=1 / \sqrt{2}, \quad\left\langle\frac{1}{2} \frac{1}{2}, \frac{1}{2}-\frac{1}{2} \mid 1,0\right\rangle=1 / \sqrt{2} \\
& \left\langle\frac{1}{2}-\frac{1}{2}, \frac{1}{2} \frac{1}{2} \mid 0,0\right\rangle=-1 / \sqrt{2}, \quad\left\langle\frac{1}{2} \frac{1}{2}, \frac{1}{2}-\frac{1}{2} \mid 0,0\right\rangle=1 / \sqrt{2} .
\end{aligned}
$$

This means that

$$
\begin{aligned}
a|2,0\rangle+\alpha|1,0\rangle+\beta|0,0\rangle & \simeq \frac{1}{\sqrt{2}} a \gamma\left|\frac{3}{2}-\frac{1}{2}, \frac{1}{2} \frac{1}{2}\right\rangle \\
+ & \frac{1}{\sqrt{2}} b(\alpha-\beta)\left|\frac{1}{2}-\frac{1}{2}, \frac{1}{2} \frac{1}{2}\right\rangle \\
& +\frac{1}{\sqrt{2}} a \gamma\left|\frac{3}{2} \frac{1}{2}, \frac{1}{2}-\frac{1}{2}\right\rangle \\
+ & \frac{1}{\sqrt{2}} b(\alpha+\beta)\left|\frac{1}{2} \frac{1}{2}, \frac{1}{2}-\frac{1}{2}\right\rangle .
\end{aligned}
$$

The first two terms correspond to the $n K^{+}$state, and the last two terms correspond to the $p K^{0}$ state. The probability ratio of the two decay modes can be expressed as

$$
\frac{\Gamma\left(\Theta^{+} \rightarrow n K^{+}\right)}{\Gamma\left(\Theta^{+} \rightarrow p K^{0}\right)}=\frac{\left\langle\Theta^{+} \mid n K^{+}\right\rangle}{\left\langle\Theta^{+} \mid p K^{0}\right\rangle}=\frac{(a \gamma)^{2}+b^{2}(\alpha-\beta)^{2}}{(a \gamma)^{2}+b^{2}(\alpha+\beta)^{2}} .
$$

As we mentioned before, $\gamma$ is very small and can be ignored, we thus get

$$
\frac{\Gamma\left(\Theta^{+} \rightarrow n K^{+}\right)}{\Gamma\left(\Theta^{+} \rightarrow p K^{0}\right)}=\frac{(\alpha-\beta)^{2}}{(\alpha+\beta)^{2}} \text {. }
$$

We still have not taken into account of the spin and parity of $\Theta^{+}$. As one of the properties of centrifugal barrier, the decay probability is directly proportional to a factor of $k^{2 L+1}$, where $k$ is the center of mass decay momentum, and $L$ is the orbital angular momentum. 24 Therefore we get the probability ratio of the two decay modes

$$
\frac{\Gamma\left(\Theta^{+} \rightarrow n K^{+}\right)}{\Gamma\left(\Theta^{+} \rightarrow p K^{0}\right)}=\frac{(\alpha-\beta)^{2}}{(\alpha+\beta)^{2}}\left(\frac{k_{1}}{k_{2}}\right)^{2 L+1},
$$

with general symmetry consideration of isospin, spin, and parity taken into account.

In this paper we adopt the $\Theta^{+}$mass $m_{\Theta^{+}}=1541 \mathrm{MeV}$, so we get $k_{1}=283.1 \mathrm{MeV}$ for the $\Theta^{+} \rightarrow n K^{+}$decay mode and $k_{2}=279.6 \mathrm{MeV}$ for the $\Theta^{+} \rightarrow p K^{0}$ decay mode. The spin and parity of $p$ and $n$ are $J^{P}=\frac{1}{2}^{+}$, of $K^{0}$ and $K^{+}$are $J^{P}=0^{-}$. Then the total angular momentum of final state is $\frac{1}{2}+L$, and parity is $(-1)^{L+1}$, where $L$ is the orbital angular momentum. The possible spin and parity of $\Theta^{+}(u u d d \bar{s})$ may 
be $\frac{1}{2}^{-}, \frac{1}{2}^{+}, \frac{3}{2}^{+}, \frac{3}{2}^{-}, \frac{5}{2}^{-}, \frac{5}{2}^{+}, \cdots$. Because of parity conservation, the corresponding orbital angular momentum of decay modes are $0,1,1,2,2,3, \cdots$. Higher spin states seem unlikely for the ground state pentaquark, so we do not calculate them further. We thus obtain Table 1 for the probability ratio of the two decay modes for the $\Theta^{+}$ state with different spin and parity.

Table 1. The decay probability ratio of the two decay modes for the $\Theta^{+}$state with different spin and parity.

\begin{tabular}{ccc}
\hline$J^{P}$ & $\mathrm{~L}$ & $\frac{\Gamma\left(\Theta^{+} \rightarrow n K^{+}\right)}{\Gamma\left(\Theta^{+} \rightarrow p K^{0}\right)}$ \\
\hline$\frac{1}{2}^{-}$ & 0 & $\frac{(\alpha-\beta)^{2}}{(\alpha+\beta)^{2}} \cdot 1.013$ \\
\hline$\frac{1}{2}^{+}$ & 1 & $\frac{(\alpha-\beta)^{2}}{(\alpha+\beta)^{2}} \cdot 1.038$ \\
\hline$\frac{3}{2}^{+}$ & 1 & $\frac{(\alpha-\beta)^{2}}{(\alpha+\beta)^{2}} \cdot 1.038$ \\
\hline$\frac{3}{2}^{-}$ & 2 & $\frac{(\alpha-\beta)^{2}}{(\alpha+\beta)^{2}} \cdot 1.064$ \\
\hline$\frac{5}{2}^{-}$ & 2 & $\frac{(\alpha-\beta)^{2}}{(\alpha+\beta)^{2}} \cdot 1.064$ \\
\hline$\frac{5}{2}^{+}$ & 3 & $\frac{(\alpha-\beta)^{2}}{(\alpha+\beta)^{2}} \cdot 1.091$ \\
\hline
\end{tabular}

From Table 1, we can find that different spin and parity give little difference to the probability ratio of the two decay modes, as the difference between the center of mass decay momenta $k_{1}$ and $k_{2}$ is very small. The results in Table 1 are valid for the $\Theta^{+}$pentaquark to be a pure isoscalar $(I=0)$ and isovector $(I=1)$ state, or a mixing isoscalar and isovector state, or an isotensor state with mixture of isoscalar and isovector components, as they are corresponding to the specific situation of $a=0, \alpha=0, \beta=1$ for isoscalar, or $a=0, \alpha=1, \beta=0$ for isovector, or $a=0$ with nonzero $\alpha$ and $\beta$ for isoscalar and isovector mixture, or $a<1$ with small $\alpha$ and $\beta$ for isotensor with mixing of isoscalar and isovector. Thus our result of the probability ratio of the two decay modes is applicable to general situations.

It is claimed 16 that the SAPHIR experiment suggests an isospin of $I=0$ for the observed $\Theta^{+}$state from the absence of a signal in the $p K^{+}$invariant mass distribution in the $\gamma p \rightarrow p K^{+} K^{-}$process. We need to examine the situation carefully, provided that the production mechanism of $\Theta^{+}$is not known yet. In photoproduction of pentaquark $\Theta$ states on the nucleon, we assume that the production is through the $K^{0} \overline{K^{0}}$ and $K^{+} K^{-}$components of the photon. The spin, parity, and $\mathrm{C}$-parity for this component of the photon should be $J^{P C}=1^{--}$. From vector dominance mode 25 we know that the interaction of the photon (virtual photon) can be effectively described by the vector mesons $(V=\rho, \omega, \phi$ with quantum numbers $\left.J^{P C}=1^{--}\right)$which have both $I=0$ and 1 states. From the available particle listing 26 we notice that the $I=0$ mesons ( $\phi$ 's) can decay into $K \bar{K}$, whereas the $K \bar{K}$ decay ratio of $I=1$ mesons ( $\rho$ 's) is very small. This implies that the $I=0$ component of the photon contains the $s \bar{s}$ content that may contribute to $\Theta$ production 
via strong interaction. From this picture, the production of $\Theta$ 's in photoproduction process is through $\gamma p \rightarrow K^{0} \overline{K^{0}} p \rightarrow \Theta^{+} \overline{K^{0}}$ and $\gamma p \rightarrow K^{+} K^{-} p \rightarrow \Theta^{++} K^{-}$ on the proton, and $\gamma n \rightarrow K^{+} K^{-} n \rightarrow \Theta^{+} K^{-}$and $\gamma n \rightarrow K^{+} K^{-} n$ without $\Theta^{++}$ on the neutron. Thus the only process that can contributes to $\Theta^{++}$production is $\gamma p \rightarrow \Theta^{++} K^{-}$. We assume that the $\Theta^{++}$state, which belongs to the isospin pentaplet $\Theta=\left(\Theta^{-}, \Theta^{0}, \Theta^{+}, \Theta^{++}, \Theta^{+++}\right)$, is of isospin $\left(I, I_{z}\right)=(2,1)$. The isospin of initial state $|i\rangle=|\gamma p\rangle$ is $|0,0\rangle|1 / 2,1 / 2\rangle$, whereas the isospin of final state $|f\rangle=\left|\Theta^{++} K^{-}\right\rangle$is $|2,1\rangle|1 / 2,-1 / 2\rangle$, from which we find that the amplitude $\langle f \mid i\rangle$ cancels exactly. However, the amplitude of $\Theta^{+}$production is also zero if $\Theta^{+}$is an isotensor; which is in contradiction with the experimental observation. If $\Theta^{+}$and $\Theta^{++}$are isovector partners, both the amplitudes of $\Theta^{+}$and $\Theta^{++}$productions are non-zero and of the same order. Thus the observation of no $\Theta^{++}$peak in the photoproduction on the nucleon can exclude both the $I=1$ and $I=2$ cases, if the mechanism of $\Theta$ production is through the isoscalar component of the photon as we suggested.

If the $I=1$ component of electromagnetic current (i.e., the photon) contributes dominantly to the production of pentaquark $\Theta$ 's, as some literature assumed, 1916 we now show that the absence of no $\Theta^{++}$peak cannot rule out an isovector $\Theta^{+}$. We assume that the $\Theta^{++}$state, which belongs to the isospin triplet $\Theta=\left(\Theta^{0}, \Theta^{+}, \Theta^{++}\right)$, is of isospin $\left(I, I_{z}\right)=(1,1)$. The isospin of initial state $|i\rangle=|\gamma p\rangle$ is $|1,0\rangle|1 / 2,1 / 2\rangle$, whereas the isospin of final state $|f\rangle=\left|\Theta^{++} K^{-}\right\rangle$is $|1,1\rangle|1 / 2,-1 / 2\rangle$. From ClebschGordan coefficients we have

$$
\begin{aligned}
& |i\rangle=|1,0\rangle\left|\frac{1}{2}, \frac{1}{2}\right\rangle=\sqrt{\frac{2}{3}}\left|\frac{3}{2}, \frac{1}{2}\right\rangle-\sqrt{\frac{1}{3}}\left|\frac{1}{2}, \frac{1}{2}\right\rangle ; \\
& |f\rangle=|1,1\rangle\left|\frac{1}{2},-\frac{1}{2}\right\rangle=\sqrt{\frac{1}{3}}\left|\frac{3}{2}, \frac{1}{2}\right\rangle+\sqrt{\frac{2}{3}}\left|\frac{1}{2}, \frac{1}{2}\right\rangle,
\end{aligned}
$$

from which we find that the amplitude $\langle f \mid i\rangle$ cancels exactly. Thus the observation of no $\Theta^{++}$peak in the photoproduction on the nucleon can not rule out the $I=1$ case for $\Theta^{+}$, but the $I=2$ case in this situation. The pentaquark $\Theta^{+}$state could be thus pure $I=0$ state, or $I=0$ state with small mixture of $I=1$ component, or $I=1$ state with small mixture of $I=0$ component if the isovector part of the photon contributes dominantly to the $\Theta^{+}$production. Therefore our results of decay probability ratio may provide useful information to constrain the isospin of the $\Theta^{+}$ state.

Though Nature permits the existence of isospin mixing state (for example, the photon is an $I=0,1$ mixing state), approximate isospin conservation in the strong interactions implies that the mass matrix of the strongly interacting particles will approximately commute with the isospin generators, and thus that the mass eigenstates will be approximate isospin eigenstates. This means that the most likely possibilities should be $\alpha \approx 1$ with small $\beta$, or $\beta \approx 1$ with small $\alpha$ if the production mechanism of $\Theta^{+}$is through the $I=1$ component of photon as most literature suggested. From our above argument for an important isoscalar contribution of the photon to $\Theta$ production, we suggest that the observed $\Theta^{+}$is likely an isoscalar. It is 
thus necessary to check whether our results are still experimentally measurable for the case of a large $\alpha \approx 1$ with small $\beta$, or vise verse. Assuming that $\alpha=0.98$ and $\beta=0.02$, which is of the right order of isospin breaking for baryons, we find that $\frac{(\alpha-\beta)^{2}}{(\alpha+\beta)^{2}} \approx 0.92$, which is measurable within experimental precision provided with enough number of events in the near future. In case of a larger isospin breaking, say that $\beta \approx 0.05$, we find that $\frac{(\alpha-\beta)^{2}}{(\alpha+\beta)^{2}} \approx 0.81$. This implies that our results are also effective to reveal isospin breaking effect in $\Theta^{+}$decays, if $\Theta^{+}$is an isoscalar as most authors predicted.

It is necessary to point out that the decay ratio we discussed is based on an assumption of isospin conservation in the decay processes. There is also a suggestion 27 that the observed $\Theta^{+}$state might be a heptaquark with the overlap of a pion, a kaon, and a nucleon. In this case, the decay ratio of two decay modes may differ from the prediction in our work. It is also possible that the decays are not through strong interaction, or other isospin violating mechanism may involve. The decay ratio predicted in this work is thus helpful to reveal detailed properties of the observed $\Theta^{+}$state, and to confirm the observed $\Theta^{+}$state as being of pentaquark $u u d d \bar{s}$ configuration. From another point of view, there may be higher $\Theta^{*+}(u u d d \bar{s})$ state beyond the anti-decuplet $\{\overline{10}\}$ multiplet. ${ }^{2829}$ It has been speculated from QCD sum rules that the isoscalar, isovector, and isotensor states of $\Theta^{+}$and $\Theta^{*+}$ pentaquarks may lie close to each other ${ }^{30}$ The results in this work are applicable to these $\Theta^{*+}(u u d d \bar{s})$ states if the mass difference is considered.

In summary, the decay probability ratio of the two decay modes $\Theta^{+} \rightarrow n K^{+}$ and $\Theta^{+} \rightarrow p K^{0}$ for the pentaquark $\Theta^{+}$state is examined with general symmetry consideration of isospin, spin, and parity. This ratio depends on two coefficients $\alpha$ and $\beta$ which can represent a pure isoscalar or isovector state, or an isoscalar and isovector mixing state, or an isotensor state with mixture of isoscalar and isovector components. The dependence on spin and parity of the pentaquark $\Theta^{+}$state is also considered, and the effect is found to be small due to small difference between the center of mass decay momenta of the two decay modes. We also provided an analysis on the constraint of the isospin of $\Theta^{+}$from the absence a peak in the $p K^{+}$invariant mass distribution in the $\gamma p \rightarrow p K^{+} K^{-}$process. Future experimental results about the decay probability ratio may confirm and provide information about the properties of the pentaquark $\Theta^{+}$state.

\section{Acknowledgements}

We acknowledge the helpful discussions with Kuang-Ta Chao, Yanlin Ye, Chuan Liu, Xiaorui Lu, Weilin Yu, Shi-Lin Zhu, K. Maltman, and H.E. Jackson. This work is partially supported by National Natural Science Foundation of China under Grant Numbers 10025523, 10145008 and 90103007.

1. M. Gell-Mann, Phys. Lett. 8 (1964) 214; G. Zweig, Preprint CERN-8182/Th.401 (Jan. 1964), unpublished; and Preprint CERN-8419/Th.412 (Feb. 1964), unpublished. 
2. R.L. Jaffe, Phys. Rev. D 15 (1977) 281; Proc. Topical Conference on Baryon Resonances, Oxford, July 1976, SLAC-PUB-1774.

D. Strottman, Phys. Rev. D 18 (1978) 2716; 20 (1979) 748.

3. C. Gignoux, B. Silvestre-Brac, J.M. Richard, Phys. Lett. B 193 (1987) 323; H.J. Lipkin, Phys. Lett. B 195 (1987) 484.

4. H. Gao, B.-Q. Ma, Mod. Phys. Lett. A 14 (1999) 2313.

5. H.J. Lipkin, Nucl. Phys. A 625 (1997) 207.

6. T.H.R. Skyrme, Proc. Roy. Soc. A 260 (1961) 127.

7. E. Witten, Nucl. Phys. B 223 (1983) 422 and 433.

8. A. V. Manohar, Nucl. Phys. B 248 (1984) 19; M. Chemtob, Nucl. Phys. B 256 (1985) 600.

See, also, M.-L. Yan, X.-H. Meng, Commun. Theor. Phys. 24 (1995) 435.

9. M. Praszalowicz, in Skyrmions and Anomalies ( M. Jezabek and M. Praszalowicz, eds.), World Scientific (1987), 112-131;

M. Praszalowicz, Phys. Lett. B 575 (2003) 234.

10. H. Walliser, Nucl. Phys. A 548 (1992) 649.

11. D. Diakonov, V. Petrov, M. Polyakov, Z. Phys. A 359 (1997) 305.

12. H. Weigel, Eur. Phys. J. A 2 (1998) 391.

13. T. Nakano, et al., Phys. Rev. Lett. 91 (2003) 012002.

14. DIANA Collaboration, V.V. Barmin, et al., hep-ex/0304040 Phys. Atom. Nucl. 66, 1715 (2003).

15. CLAS Collaboration, S. Stepanyan, et al., Phys. Rev. Lett. 91 (2003) 252001.

16. SAPHIR Collaboration, J. Barth, et al., Phys. Lett. B 572 (2003) 127.

17. HERMES Collaboration, A. Airapetian, et al., Phys. Lett. B 585 (2004) 213.

18. In CLAS experiment, an additional $p$ is also measured in the exclusive $\gamma D \rightarrow$ $K^{-} p\left(\Theta^{+} \rightarrow K^{+} n\right)$ process, just as the $\gamma^{*} D \rightarrow K^{-} p \Theta^{+}$process suggested in Ref. 4

19. S. Capstick, P.R. Page, and W. Roberts, Phys. Lett. B 570 (2003) 185.

20. R. Jaffe, F. Wilczek, Phys. Rev. Lett. 91 (2003) 232003.

21. N. Isgur, Phys. Rev. D 21 (1980) 779 [Erratum-ibid. D 23 (1981) 817].

22. G.A. Miller, Phys. Rev. C 57 (1998) 1492.

23. G. Karl, Phys. Lett. B 328 (1994) 149 [Erratum-ibid. B 341 (1995) 449].

24. C.S. Gao, J.Y. Zeng, Lecture on Particle Physics and Nuclear Physics (in Chinese), Higher Education Press (1990), 101.

25. See, Eletromagnetic Interactions of Hadrons, Volume I and II, edited by A. Donnachie and G. Shaw, 1978 Plenum Press, New York.

26. Particle Data Group, K. Hagiwara et al., Phys. Rev. D 66 (2002) 010001.

27. P. Bicudo, G.M. Marques, Phys. Rev. D 69 (2004) 011503(R).

28. H. Walliser, V.B. Kopeliovich, J. Exp. Theor. Phys. 97 (2003) 433 (hep-ph/0304058);

D. Borisyuk, M. Faber, A. Kobushkin, hep-ph/0307370

29. B. Wu and B.-Q. Ma, Phys. Rev. D 60 (2004) 077501; Phys. Lett. B 586 (2004) 62.

30. S.-L. Zhu, Phys. Rev. Lett. 91 (2003) 232002. 